\title{
Identification of a Genetic Alteration in the Code for Bilirubin UDP-Glucuronosyltransferase in the UGT1 Gene Complex of a Crigler-Najjar Type I Patient
}

\author{
Joseph K. Ritter," Matthew T. Yeatman," Patrick Ferreira, ${ }^{*}$ and Ida S. Owens* \\ *Human Genetics Branch, National Institute of Child Health and Human Development, National Institutes of Health, Bethesda, \\ Maryland 20892; ${ }^{\ddagger}$ Division of Medical Genetics, University of Alberta, Edmonton, Alberta T6G $2 B 7$ Canada
}

\begin{abstract}
Patients with Crigler-Najiar syndrome (CN) type I inherit an autosomal recessive trait for hyperbilirubinemia, which is characterized by the total absence of bilirubin UDP-glucuronosyltransferase (transferase) activity. The recent identification of two bilirubin transferase isoforms with identical carboxyl termini (Ritter, J. K., J. M. Crawford, and I. S. Owens. 1991. J. Biol. Chem. 266:1043-1047) led to the discovery of a unique locus, UGT1, which encodes a family of UDP-glucuronosyltransferase isozymes, including the two bilirubin forms (Ritter, J. K., F. Chen, Y. Y. Sheen, H. M. Tran, S. Kimura, M. T. Yeatman, and I. S. Owens. 1992. J. Biol. Chem. 267:32573261). The $U G T 1$ locus features a complex of six overlapping transcriptional units encoding transferases, each of which shares the four most $3^{\prime}$ exons $\left(2,3,4\right.$, and 5) specifying the $3^{\prime}$ half of the transferase coding regions (codons 289-533) and the entire $3^{\prime}$ untranslated region of each mRNA. This gene model predicts that a single critical mutation in any of these four "common" exons may inactivate the entire family of encoded transferases. In agreement with this prediction, we show here that in the first CN type I individual analyzed (patient F.B.), a 13-bp deletion has occurred in exon 2 . Analysis of product generated by the polymerase chain reaction and genomic DNA demonstrated that F.B. is homozygous for the defective allele ( $\left.U G T 1^{*} \mathrm{FB}\right)$, and that the consanguineous parents are both heterozygotic at this locus. The mutation is predicted to result in the synthesis of severely truncated bilirubin transferase isozymes that are lacking a highly conserved sequence in the carboxyl-terminus and the characteristic membrane (endoplasmic reticulum)-anchoring segment of the protein molecule. (J. Clin. Invest. 1992.90:150-155.) Key words: bilirubin - UDP-glucuronosyltransferase $\bullet$ hyperbilirubinemia $\bullet$ genetic defect $\bullet$ deletion
\end{abstract}

\section{Introduction}

Bilirubin, a toxic metabolite formed by the ongoing turnover of hemoproteins (most significantly hemoglobin) is normally metabolized to excretable derivatives by conjugation of one or both of the carboxyl groups of its propionyl side chains (C-8 or $\mathrm{C}-12$ ) with glucuronic acid (1). Both the formation of the C-8 or $\mathrm{C}-12$ monoglucuronide or the $\mathrm{C}-8, \mathrm{C}-12$ diglucuronide is catalyzed by hepatic bilirubin UDP-glucuronosyltransferase

Address correspondence to Dr. Ida S. Owens, 9000 Rockville Pike, Bldg. 10, NIH, Room 9S-242, Bethesda, MD 20892

Received for publication 23 September 1991 and in revised form 27 January 1992.

The Journal of Clinical Investigation, Inc.

Volume 90, July 1992, 150-155
(2). Inadequate bilirubin glucuronidation, commonly observed in neonates (3), breast-fed infants (4), and as a result of liver diseases (5), is associated with the accumulation of serum bilirubin and the appearance of the clinical features of jaundice (3). Coincident with the continuous and marked elevations ( $>340$ $\mu \mathrm{mol} /$ liter) in serum bilirubin concentrations seen in serious diseased states is the risk of kernicterus, the degenerative changes associated with bilirubin deposition in the brain (3). Milder elevations in the serum level of this heme derivative are seen in approximately half of all neonates, and are due to delays in the developmental onset of bilirubin transferase activity normally occurring one to five days after birth. Although the short-term maintenance of jaundiced neonates is a major concern of pediatric clinicians, children with inborn errors in bilirubin glucuronidation (totally lacking this function) present an even greater challenge. In 1952, Crigler and Najjar (6) described a syndrome featuring chronic nonhemolytic unconjugated hyperbilirubinemia in which hepatic bilirubin UDPglucuronosyltransferase activity is absent. The disorder, Crigler-Najjar syndrome (CN) 1 type $\mathrm{I}$, is inherited in an autosomal recessive pattern (7-9). The fatalities associated with this condition confirm the need to detoxify bilirubin. Therefore, the characterization of the bilirubin transferase gene is critical to making progress in the development of therapies for the type I disease.

Recently, we reported the isolation and characterization of two human liver bilirubin UDP-glucuronosyltransferase cDNAs, HUG-Br1 and HUG-Br2 (2), which, upon expression individually in COS- 1 cells, were both found to encode isoforms that catalyze the formation of the two bilirubin monoglucuronides and the diglucuronide. Northern analysis of HUG-Brl and HUG-Br2 mRNA showed that HUG-Brl is fourfold more abundant than HUG-Br2, and that in the $\mathrm{Er}$ ythrocebus patas monkey, mRNA hybridizing with $\mathrm{HUG}-\mathrm{Br} 2$ but not HUG-Brl is inducible by phenobarbital. We also showed (2) that mRNAs corresponding to both bilirubin transferase isoforms were present in the explanted liver of a $\mathrm{CN}$ type I patient (not F.B.). The observations that the mRNAs were of the normal size $(\sim 2.6 \mathrm{~kb})$ and abundance suggest that neither defective regulation nor gross abnormalities in gene structure is causative, and that smaller point mutations are probably involved.

In further studies, we isolated and determined the structure of an 85-kb locus (10), UGT1, on chromosome 2 (11), which encodes the two bilirubin UDP-glucuronosyltransferase isoforms. With this information, we began the search for mutations responsible for the lack of detectable bilirubin transferase

1. Abbreviations used in this paper: $\mathrm{CR}$, Crigler-Najjar syndrome; $\mathrm{PCR}$, polymerase chain reaction. 
activity in $\mathrm{CN}$ type I patients. Here we show that in the first affected individual studied in detail, a critical 13-bp deletion exists in exon 2 of the $U G T 1$ gene complex. The predicted effect of the mutation on the primary structure of the bilirubin transferase isoenzymes, together with our demonstration of the expected familial distribution of the defective allele, is described in this report.

\section{Methods}

CN type I human samples. Blood samples were collected from F.B., a 4-yr-old male $\mathrm{CN}$ type I patient, his parents, and A.R., an unrelated normal female. The patient is the 37-wk, 3,470-g offspring of a consanguineous mating of Italian descent. All other immediate family members are clinically normal. Marked jaundice appeared at $12 \mathrm{~d}$ of age, and peak bilirubin (all unconjugated) was $598 \mu \mathrm{mol} / \mathrm{liter}$. Hemoglobin was $19.6 \mathrm{~g} \%$, and the reticulocyte count was $0.7 \%$. The mother was $\mathrm{O}$ negative, and the baby was $\mathrm{O}$ positive, but direct and indirect Coombs' tests were negative. The results of the following studies were normal: urinary menthol glucuronides after oral administration (8), transaminases, alkaline phosphatase, 5'-nucleotidase, total bile acids, thyroid functions tests, prothrombin time, partial thromboplastin time, glucose-6-phosphate-dehydrogenase screen, pyruvate kinase screen, $\alpha$-antitrypsin levels, and various bacterial cultures. Unconjugated hyperbilirubinemia persisted despite repeated exchange transfusions, phototherapy, and administration of phenobarbital. A liver biopsy at $6 \mathrm{wk}$ of age confirmed normal histology and the absence of bilirubin UDP-glucuronosyltransferase activity (12). Bilirubin glucuronides were absent in duodenal bile, serum, and urine (13). Treatment with whole body phototherapy $(12 \mathrm{~h} / \mathrm{d})$ and cholestyramine $(3 \mathrm{~g} / \mathrm{d})$ was initiated. Under this treatment regimen, bilirubin levels ranged between $300-450 \mu \mathrm{mol} / \mathrm{liter}$. At age $4 \frac{1}{2} \mathrm{yr}$ he showed normal somatic, intellectual, and neurological development.

Preparation of lymphocyte genomic DNA. The blood samples were transferred to $50-\mathrm{ml}$ Falcon tubes and spun at $4,000 \mathrm{rpm}$ for $30 \mathrm{~min}$ in an HS-4 rotor (Dupont Instruments-Sorvall Biomedical Div., Wilmington, DE). The pellet was resuspended in $40 \mathrm{ml}$ of prechilled buffer (10 mM Tris-Cl, pH 7.5, $10 \mathrm{mM} \mathrm{NaCl}, 3 \mathrm{mM} \mathrm{MgCl}$, and $0.2 \%$ Triton $\mathrm{X}-100$ ), recentrifuged, and the step was repeated a second time. The pellet was resuspended the final time in $2 \mathrm{ml}$ of SDS solution $(10 \mathrm{mM}$ Tris-Cl, pH 7.5, $20 \mathrm{mM} \mathrm{NaCl}, 5 \mathrm{mM} \mathrm{MgCl}$, and 2\% SDS). Proteinase $\mathrm{K}$ $(900 \mu \mathrm{g} / 10 \mathrm{ml}$ vol blood) was added, and the tubes were incubated at $37^{\circ} \mathrm{C}$ for $2 \mathrm{~h}$ and then at room temperature overnight. The samples were then extracted five times with phenol/chloroform (1:1) and dialyzed at $4^{\circ} \mathrm{C}$ overnight against $10 \mathrm{mM}$ Tris, $\mathrm{pH} 7.5,1 \mathrm{mM}$ EDTA with several changes.

Oligonucleotide primer synthesis. Oligonucleotides for PCR amplification of the four $U G T 1$ common exons were synthesized using a Cyclone DNA synthesizer, (model 8400, MilliGen/Biosearch, Bedford, MA) and a 200-coupling $\beta$-phosphoamidite kit (MilliGen/Biosearch). The oligonucleotides and their sequences are as follows: PXG3, 5'CTATCTCAAACACGCATGCC-3'; PXG4, 5'-GGATTAGCGCTCCTGTGAA-3'; PXG5, 5'-GTCTTTCTTTACGTTCTGCTC-3'; PXG6, 5'-GACCCTGGTTTGACCTATAC-3'; PXG7, 5'-CTCAGAGATGTAACTGCTGAC-3'; PXG8, 5'-CATGAATGCCATGACCAAAG-3'; PXG9, 5'-GTTCATACCACAGGTGTTCCA-3'; PXG10, 5'-GGAAATGACTAGGGAATGGTTC-3'; J127,5'-TCTGAGACCATTGATCC$3^{\prime}$. After synthesis, the oligos were cleaved and deprotected with concentrated aqueous ammonia (29\%), as suggested by the manufacturer, dried and resuspended in sterile water, reprecipitated with ethanol, and resuspended in $10 \mathrm{mM}$ Tris, $\mathrm{pH} 8$, and $1 \mathrm{mM}$ EDTA. Oligos were analyzed by electrophoresis through a $12 \%$ polyacrylamide gel.

Polymerase chain reaction, subcloning, and sequencing of $P C R$ products. In order to localize mutations in the $U G T 1$ locus of $\mathrm{CN}$ patients, the polymerase chain reaction (PCR) was performed such that each of the four common exons was amplified in an individual reaction. Nucleotide sequencing of the $U G T 1$ gene (10) permitted the iden- tification and synthesis of oligonucleotide primers binding to sequences in the region immediately $5^{\prime}$ (sense primer) or $3^{\prime}$ (antisense primer) to each exon. Using normal DNA, primers PXG3 and PXG4 amplify a 311-bp fragment containing the complete 132-bp exon 2 plus $125 \mathrm{bp}$ and $54 \mathrm{bp}$ of $5^{\prime}$ and $3^{\prime}$ flanking sequence, respectively; primers PXG5 and PXG6 amplify a 164-bp product containing the complete 88-bp exon 3 plus $36 \mathrm{bp}$ and $40 \mathrm{bp}$ of $5^{\prime}$ and $3^{\prime}$ adjacent sequence, respectively; primers PXG7 and PXG8 amplify a 309-bp fragment containing the complete 220-bp exon 4 plus 45 bp and 44 bp, respectively, of the adjacent $5^{\prime}$ and $3^{\prime}$ introns; and primers PXG9 and PXG10 amplify a 398-bp fragment corresponding to the first 349 bp of exon 5 (segment containing the final part of the open reading frame and translation stop codon) plus $49 \mathrm{bp}$ of its $5^{\prime}$ flanking sequence.

Each primer set was used in standard PCR reaction mixtures that contained genomic DNA $(0.1 \mu \mathrm{g})$, each of the designated primer combinations ( $1 \mu \mathrm{M}$ each), $10 \mathrm{mM}$ Tris- $\mathrm{HCl}, \mathrm{pH} 8.3\left(\right.$ at $\left.25^{\circ} \mathrm{C}\right), 50 \mathrm{mM} \mathrm{KCl}$, $1.5 \mathrm{mM} \mathrm{MgCl}, 0.001 \%$ (wt/vol) gelatin (cat. no. G2500, Sigma Chemical Co., St. Louis, MO), $0.2 \mathrm{mM}$ each dNTP, and $0.25 \mathrm{U} / \mathrm{ml}$ of Amplitaq polymerase. Reaction mixes were overlaid with mineral oil $(50 \mu \mathrm{l})$ and subjected to 30 cycles at $94^{\circ} \mathrm{C}$ for $1 \mathrm{~min}, 56^{\circ} \mathrm{C}$ for $1.5 \mathrm{~min}$, and $72^{\circ} \mathrm{C}$ for $3 \mathrm{~min}$ using a thermocycler (Perkin-Elmer Cetus Corp., Emeryville, CA). The final extension step at $72^{\circ} \mathrm{C}$ lasted $30 \mathrm{~min}$. Using normal DNA, these primer combinations result in the amplification of fragments which are $311,164,309$, and 398 bp in length for exons 2-5, respectively. PCR products were polished with Klenow, phosphorylated with T4 polynucleotide kinase, and subjected to electrophoresis through $1 \%$ low melting point agarose. Bands were visualized with ethidium bromide, excised, and blunt-end ligated with Smal-cleaved and calf intestinal alkaline phosphatase-dephosphorylated pBluescript II SK $+(0.1 \mu \mathrm{g})$ using T4 DNA ligase (1 U). After incubation at $16^{\circ} \mathrm{C}$ for $18 \mathrm{~h}$, the solidified agarose ligation reactions were melted, diluted 10-fold with RF2 buffer (14), and $50 \mu \mathrm{l}$ was used to transform $100 \mu \mathrm{l}$ of frozen competent Escherichia coli strain XL1-Blue (Stratagene Inc., La Jolla, CA) prepared using protocol 3 with the rubidium-containing buffers RF1 and RF2 (14). Ampicillin-resistant transformants were cultured in $7 \mathrm{ml}$ of Luria broth that contained ampicillin $(100 \mu \mathrm{g} / \mathrm{ml})$, and DNA was purified and sequenced as previously described (15). Each sample was sequenced in its entirety in both directions using the Bluescript primers KS and SK (Stratagene Inc.). Two or more of each subclone were sequenced.

Materials. Amplitaq polymerase was from Cetus Corp., T4 polynucleotide kinase from Bethesda Research Laboratories (Gaithersburg, $\mathrm{MD}$ ), and the large Klenow fragment from U.S. Biochemical (Cleveland, $\mathrm{OH}$ ). All restriction endonucleases were from either New England Biolabs (Beverly, MA), Pharmacia LKB Biotechnology Inc. (Piscataway, NJ), or Bethesda Research Laboratories. Calf intestinal alkaline phosphatase was from Boehringer Mannheim Corp. (Indianapolis, IN). The vector pBluescript was purchased from Stratagene Inc.

\section{Results}

The UGTI locus. Typical liver microsomal bilirubin UDPglucuronosyltransferase activity is necessarily composed of at least two distinct isozymes which we now appreciate from an earlier demonstration of the presence of two different isoelectrifocusing activities in rat liver (16) and recently, from the demonstration of two human bilirubin transferase cDNAs, HUG-Br1 and HUG-Br2 (2), which are generated from the $U G T 1$ locus (10). UGTI is a unique gene complex featuring nested transferase transcriptional units (Fig. $1 A$ ). As demonstrated (10), each of these units, UGTIA-UGTIF, specifies a unique transferase isoform. The complex contains a tandem array of six promoters (Fig. $1 \mathrm{~A}$, arrows) with each positioned adjacent to a first exon (Fig. $1 A$, designated boxes, $\sim 880-960$ bp each), thereby defining the transcriptional start site for the unit. At the $3^{\prime}$ end of the locus are four exons (Fig. $1 A, 2-5$ ) that 
are used by all six transcriptional units. Primary messenger RNA transcripts (not shown) are predicted by each unit (10), which undergo differential splicing to produce mature RNAs containing different $5^{\prime}$ termini but identical $3^{\prime}$ termini (Fig. $1 B$ ) $\sim 2.6 \mathrm{~kb}$ in length. Two of these encoded isoforms, UGTIA and $U G T 1 D$, respectively, represent the transcriptional units that specify mRNAs corresponding to HUG-Brl and HUG$\mathrm{Br} 2$ bilirubin transferase cDNAs prepared from $\mathrm{mRNA}$ isolated from normal human liver (2). A third unit, $U G T 1 F$, encodes a phenol-specific isoform for which a corresponding cDNA has also been identified (17). The existence of mRNAs encoded by $U G T 1 B P$ and $U G T 1 C$ (but not $U G T 1 E$ ) was confirmed by PCR analysis of a single human liver RNA sample (10). Because the model predicts that critical mutations introduced into any of the common exons 2-5 will result in the inactivation of all encoded isoforms, we examined these exons for differences in nucleotide sequence.

Identification of 13-bp deletion in exon 2. Exons 2-5 were amplified individually using the PCR and subcloned as described in Methods. Analysis of exon 3, 4, and 5 subclones showed that each had the normal sequence (data not shown). However, a 13-bp deletion (Fig. $1 \mathrm{~A}$, designation of gene location of defect by opened arrowhead, and Fig. 2, details of defect) was observed in each of the four independent exon 2 subclones. To confirm the mutation, four additional exon 2 subclones were tested from a second PCR reaction, indicating that the mutation was not because of errors by Taq polymerase. The deletion is located $12 \mathrm{bp}$ (downstream) from the intron 1-exon 2 junction (Fig. 2), and causes a change in the deduced amino acid sequence after residue 293 (Fig. $2 \mathrm{~A}$ ). The dark vertical line by the nucleotides of the normal DNA sequences represents the deleted bases in the DNA of the type I patient. The presence of a normal intronic branchpoint preceding exon 2 , as well as its accompanying acceptor and donor splice sites suggests there

\section{The UGT1 locus}

A

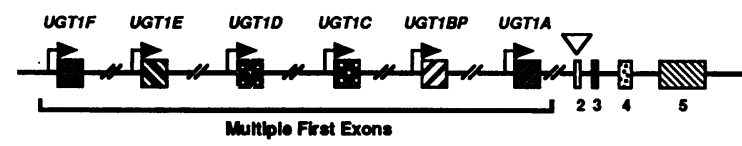

B

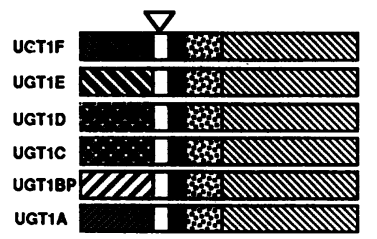

Figure 1. Schematic diagram of $U G T 1$ gene locus. $(A)$ Exon/intron arrangement of $U G T 1$. UGT1 is a complex of nested transcription units featuring multiple first exons of $U G T 1 A-U G T 1 F$ and their associated promoters (right-angled arrows), and four common exons (2-5). The solid black lines indicate intervening DNA. The complex is not drawn to scale and covers at least an $85-\mathrm{kb}$ region of chromosome 2 (11). First exons are spaced an average of $7 \mathrm{~kb}$ from each other and the four common exons are clustered in a 6-kb region at the $3^{\prime}$ end of the complex. (B) Mature RNAs produced from the $U G T 1$ gene locus. The complex encodes a family of UDP-glucuronosyltransferase mRNAs with unique $5^{\prime}$ termini (boxes with different patterns) and identical $3^{\prime}$ termini (boxes with common patterns). The products of $U G T I A$ and $U G T I D$ are the bilirubin UDP-glucuronosyltransferase encoded by HUG-Brl and HUG-Br2, respectively (2).

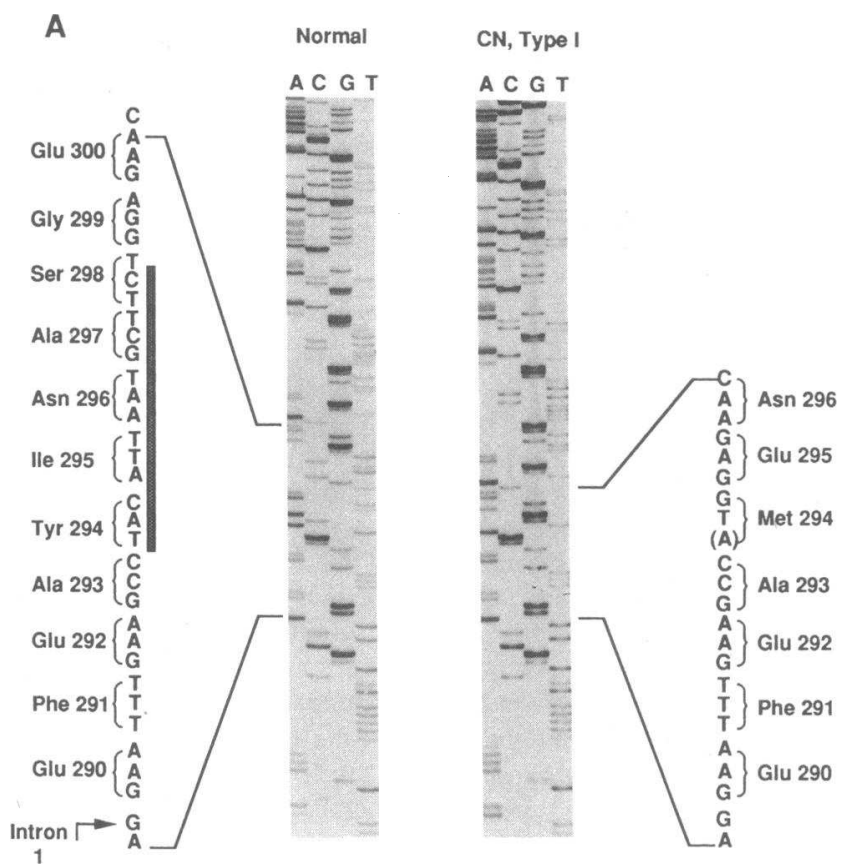

B

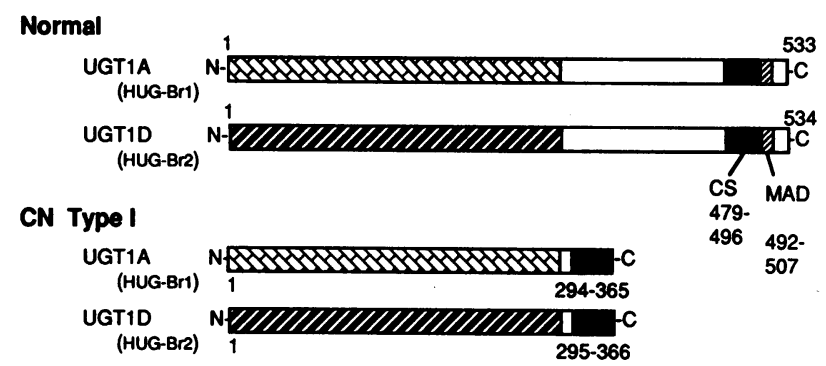

Figure 2. Autoradiograms of Sanger nucleotide sequencing reactions of normal and $\mathrm{CN}$ type I patient DNA. Plasmid DNA containing the entire $U G T 1$ exon 2 from a normal $(A)$ and the $C N$ type I patient F.B. $(B)$ was sequenced using the Bluescript primer, $\mathrm{KS}$, as described under Methods. The sequence ladders were generated by electrophoresis through a denaturing $6 \%$ polyacrylamide gel, after which the gels were transferred to blotting paper, dried, and exposed overnight to x-ray film to generate the autoradiogram shown. $A, C, T$, and $G$ are lanes corresponding to separate reactions with added dideoxy derivatives of ATP, CTP, TTP, and GTP, respectively. The boundary between the first intron and the second exon is shown. The shaded vertical line designates the 13-bp segment in normal DNA, which is deleted from the DNA of the CN type I patient. $(B)$ Predicted effect of the 13-bp deletion on the bilirubin UDP-glucuronosyltransferases encoded by $U G T 1 A$ and $U G T 1 D$. In a normal individual, the bilirubin transferases encoded by UGTIA (HUG-Br1) and UGTID (HUG-Br2) have different $\mathrm{NH}_{2}$-termini ( 288 or 289 amino acids, respectively) and identical $\mathrm{COOH}$-termini (246 amino acids). In $\mathrm{CN}$ type I patient F.B., these proteins are predicted to be severely truncated. $\mathrm{N}$ and $\mathrm{C}$, the amino and carboxyl termini of bilirubin transferase; CS (unknown function), and MAD (membrane anchoring domain) are conserved domains present in all known UDP-glucuronosyltransferases.

should be no gross effects on the level of mRNA transcription, size, or translatability.

Predicted effect of mutation on protein structure. Fig. $2 B$ shows the proposed effect of the mutation on the coding for 
bilirubin transferase. The deletion of codons 294-297 and a -1 frameshift result in the synthesis of truncated forms of both bilirubin transferase isozymes. Fig. $2 A$ shows the site leading to a change in its predicted translation. The FB sequence would, therefore, be very different from that of a normal individual after codon 294. Continuation of translation of the open reading frame (not shown in Fig. $2 \mathrm{~A}$ ) shows a TGA stop signal after the 365 th codon (illustrated schematically in Fig. $2 \mathrm{~B}$ ); thus, a 72-residue unrelated peptide is present after codon 294. Furthermore, the mutant proteins are predicted to be missing 240 residues of the transferase carboxyl terminal region which is $90 \%$ conserved between human and rat. This includes at least two recognized motifs: $(a)$ a highly conserved 18 amino acid sequence (CS, Fig. $2 \mathrm{~B}$ ) with $\geq 90 \%$ conservation among 11 known transferases (2) and references therein, and $(b)$ a mem-

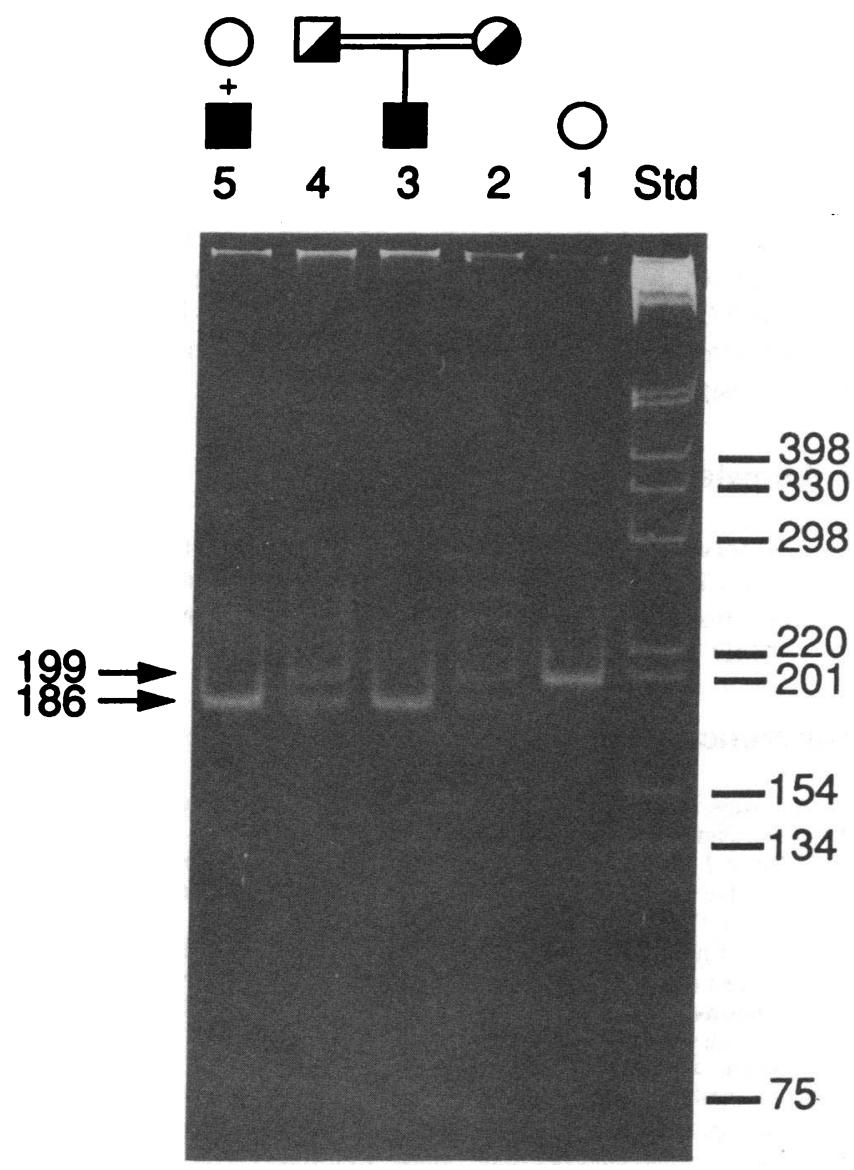

Figure 3. PCR analysis of genomic DNA from family members. Samples of PCR reactions generated from genomic DNA $(0.1 \mu \mathrm{g})$ of the $\mathrm{CN}$ type I individual, F.B. (lane 3), his parents (lanes 2 and 4), and an unrelated normal individual (lane 1) with oligonucleotides PXG3 and J127 as primers (as described in Methods) were analyzed by electrophoresis through nondenaturing polyacrylamide $(6 \%)$ for $5 \mathrm{~h}$ at $50 \mathrm{~V}$ in $0.5 \times \mathrm{TBE}$. The gel was stained with ethidium bromide, irradiated with UV, and photographed. The primers amplify a fragment representing the last $45 \mathrm{bp}$ of the first intron and the first 154 bp of exon 2. Lane 5 is a sample of a control reaction in which DNAs from F.B. (homozygous affected) and the unrelated normal (homozygous normal) were mixed in approximately equal proportions and subjected to the same PCR analysis. STD is a molecular size standard, $1 \mathrm{~kb}$ ladder (Bethesda Research Laboratories). Molecular sizes (in bp) are shown to the right of the figure. brane-anchoring domain (MAD, Fig. $2 B$ ) that permits the retention of the transferase protein in the endoplasmic reticulum membrane.

Family genetic analysis. Although it was established by a pedigree study that the parents of F.B. are consanguineous, we demonstrate the pattern of inheritance by F.B. of the defective allele. Since $\mathrm{CN}$ type $\mathrm{I}$ is an autosomal recessive disease, this suggests that F.B. is likely homozygous for the same defective allele, $U G T 1^{*} \mathrm{FB}$, and that both parents are heterozygotic at the $U G T 1^{*} \mathrm{FB}$ locus. This hypothesis was confirmed using the PCR to amplify a fragment corresponding to the last $45 \mathrm{bp}$ of intron 1 and first 154 bp of exon 2, followed by visualization of the reaction products with agarose gel electrophoresis (Fig. 3). Whereas a single band of the expected normal size (199 bp) was observed in a control sample from an unrelated normal individual (lane 1), the sample corresponding to F.B. (lane 3) contained a single band corresponding to a predicted fragment shorter by $13 \mathrm{bp}$ (186 bp). These observations are consistent with F.B. being homozygous for the allele with a 13-bp deletion. In comparison, the mother (lane 2) and father (lane 4) exhibited both the 199-bp and 186-bp bands in equal amounts. Two other slower migrating bands not seen in either the normal or CN type I case were present in the parental samples. A sample representing a reaction that included a mixture of FB and normal DNA (Fig. 3, lane 5), thereby simulating the composition of heterozygotic DNA, also exhibited these sized bands. These observations are consistent with those from other PCR studies (18) with multiple alleles, which suggest that the bands are caused by mismatched DNA strands (i.e., heteroduplexes), which migrate differently from those that are perfectly matched.

\section{Discussion}

Of the hundreds of known substrates that undergo detoxification by glucuronidation (19), bilirubin was recognized early because of the marked phenotypic changes (jaundice) associated with variable rates in its glucuronidation. Microsomes prepared from the explanted livers of patients afflicted with the ultimately fatal disease $\mathrm{CN}$ type I do not have detectable bilirubin glucuronidating activity (3), whereas activities towards most substrates are normal (see exceptions below) (20). Here we show that a $\mathrm{CN}$ type I patient contains a homozygous 13-bp deletion in common exon 2 of the gene complex $U G T 1$ encoding the two bilirubin transferase proteins. The nature of this defect is consistent with inactivation of the gene product. As deduced by translation of the altered coding region, the bilirubin transferases encoded by $U G T 1 A$ and $U G T 1 D$ are predicted to be missing the final 240 amino acids of the wild type proteins, a region which is highly conserved across species $(90 \%$ identity between rat and human) (2). This region contains two smaller domains/motifs that have been recognized as highly conserved among deduced transferase sequences including members of both major subgroups so far identified, the steroid and bilirubin/phenol subfamilies (21). A membrane-spanning domain represented by 17 consecutive hydrophobic residues normally flanked on the amino terminal side by a charged residue (Asp) and the carboxyl terminal side by a highly positively charged halt transfer signal (Lys/Arg rich), is located at the extreme carboxyl-terminus of all known transferases. The absence of this region should alter the subcellular localization of 
the $U G T 1$-encoded proteins. In addition, the highly conserved 18-residue motif overlapping the first six residues of the membrane spanning domain and present in all UDP-glucuronosyltransferase isozymes is also missing in FB. [This region was utilized as a strategy for the cloning of the human (2) and rat (22) bilirubin isoforms.] Its function is currently unknown, although it has been speculated that it participates in the binding of the common donor substrate, UDP-glucuronic acid. We have also recently shown (23) that nine amino acids between residues 385-469 in two bile acid/steroid transferase isoforms play a critical role in catalytic efficiency (i.e., whether the transferase is a high- or low-metabolizing isoform).

It should be noted that we cannot exclude the possibility that the deletion of 13 nucleotides from the 132-bp exon 2 causes defective splicing. This prospect seems unlikely since the branchpoint sequence and consensus sequences for splicing appear normal.

In addition to the predicted loss of four codons (294-297), the truncated proteins are predicted to contain a foreign 72-residue carboxyl terminus, resulting from the -1 shift in reading frame. A 1-bp deletion with a similar consequence (i.e., the introduction of a premature stop codon) was described at the cDNA levels for phenol and bilirubin transferase mRNAs of the hyperbilirubinemic Gunn rat, considered a model of $\mathrm{CN}$ type I disease (24). Iyanagi et al. (25) showed that defective 3-methylcholanthrene-induction of phenol glucuronidating activity was due to a -1 frame shift mutation at codon 414 in the mRNA for the phenol form (26) (which is the orthologue of the form encoded by the human $U G T 1 F)$. This mutation results in the synthesis of a truncated enzyme (27). More recently, Sato et al. (28) showed that the genetic defect in the bilirubin UDPglucuronosyltransferase mRNA in the Gunn rat was due to the same mutation. These data, together with our recent determination of the $U G T 1$ gene structure, provide an understanding of the complex arrangement of the locus and allow the prediction that the other forms encoded within UGT1 are also defective in the $\mathrm{CN}$ type I patient studied here, including the isozymes encoded by $U G T 1 B P, U G T 1 C$, and $U G T 1 E$. These isoforms may be involved in the glucuronidation of substrates, including phenol (17), digitoxigenin monodigitoxiside (29), 5-hydroxytryptamine $(20,30)$, and acetaminophen $(31)$, that have been associated with the jaundice locus of the rat.

Both CN type I and the hyperbilirubinemia seen in the Gunn rat, are transmitted as autosomal recessive disease entities (7-9). In humans, $\mathrm{CN}$ type I is a rare disorder which was first described in the literature in 1952 (3), and is often a consequence of consanguineous parents. As for the case described here, the heterozygosity and consanguinity of the parents are the basis of the homozygosity of F.B. at the $U G T 1^{*} F B$ locus. Using PCR, we conclusively demonstrated that both parents are carriers of the same defect seen in F.B. Whereas only a shortened band was amplifiable from the DNA of FB, bands of both the deleted size and normal size were amplifiable from that of the parents (Fig. 3). The PCR assay used in this study may be included as one in a series of diagnostic markersamong others to emerge from future characterization of the different defects associated with heritable hyperbilirubinemic disorders (e.g., CN type II or Gilbert's syndrome).

Although it is not yet known, it is unlikely that a single mutation $\left(U G T 1^{*} F B\right)$ accounts for all $\mathrm{CN}$ type I cases. The existence of multiple phenotypes associated with $\mathrm{CN}$ type I ( 9 , 32) supports the notion that multiple types of mutations in
UGTl are involved. Furthermore, van Es et al. (20) performed a biochemical and immunochemical analysis of four unrelated $\mathrm{CN}$ patients with no detectable transferase activity toward bilirubin. Whereas patients $A$ and $B$ had reduced microsomal transferase activity towards 5-hydroxytryptamine and phenol, patients $\mathrm{C}$ and $\mathrm{D}$ had normal activities toward these substrates. Furthermore, by using two different monoclonal antibodies (WP1 and HEB7) prepared against phenol and bilirubin UDPglucuronosyltransferase, van Es et al. showed that two or more immunostained isozymes were absent in patients A and B, whereas in patients $\mathrm{D}$ and $\mathrm{C}$, the polypeptide patterns were similar to controls. These results also suggest a genetic heterogeneity among $\mathrm{CN}$ type I patients; some are apparently able to synthesize full length (but presumably inactive) bilirubin transferases, whereas others (such as F.B. described here) synthesize truncated versions. From the results in this study, we are able to predict that microsomes from patient F.B. (who has not undergone liver transplant surgery and therefore has not yet been tested) will exhibit the phenotype which characterized patients $A$ and $B$. It also suggests the possibility that among $C N$ patients mutations are possible which selectively inactivate one or both of the bilirubin isoforms while sparing the remaining forms encoded by the $U G T 1$ locus (e.g., the $U G T 1 F$-encoded phenol form). It is expected that analysis of additional $\mathrm{CN}$ patients will uncover a host of different mutations; with the unique organization of the UGTI locus, the possibilities abound as to the diversity of mutational sites and their effect(s) on the function of this multiple isozyme detoxification system.

\section{Acknowledgments}

The authors gratefully acknowledge the helpful discussions of Dr. Lawrence Charnas and Dr. Janice Chou, Human Genetics Branch, National Institute of Child Health and Human Development, Bethesda, MD.

\section{References}

1. Ostrow, J. D., and N. H. Murphy. 1970. Isolation and properties of conjugated bilirubin and bile. Biochem. J. 120:311-327.

2. Ritter, J. K., J. M. Crawford, and I. S. Owens. 1991. Cloning of two human liver bilirubin UDP-glucuronosyltransferase cDNAs with expression in COS 1 cells. J. Biol. Chem. 266:1043-1047.

3. Roy Chowdhury, J., A. W. Wolkoff, and I. M. Arias. 1989. Hereditary jaundice and disorders of bilirubin metabolism. In The Metabolic Basis of Inherited Disease. C. R. Scriver, A. L. Beaudet, W. S. Sly, and D. Valle, editors. McGraw Hill Inc., New York. 1367-1408.

4. Foliot, A., J. P. Ploussard, E. Housett, B. Christoforov, R. Luzeau, and M. Odievre. 1976. Breast milk jaundice: in vitro inhibition of rat liver bilirubin-uridine diphosphate glucuronyl transferase activity and $\mathrm{Z}$ protein-bromosulfophthalein binding by human breast milk. Pediatr. Res. 10:594-598.

5. Bloomer, J. R., P. D. Berk, and R. B. Howe. 1974. Hepatic clearance of unconjugated bilirubin in cholestatic liver diseases. Am. J. Dig. Dis. 19:9-14.

6. Crigler, J. F., and V. A. Najjar. 1952. Congenital familial non-hemolytic jaundice with kernicterus. Pediatrics. 10:169-180.

7. Childs, B., J. B. Sidbury, and C. J. Migeon. 1959. Glucuronic acid conjugation by patients with familial non-hemolytic jaundice and their relatives. Pediatrics. 23:903.

8. Szabo, L., and P. Ebrey. 1963. Studies on the inheritance of Crigler-Najjar syndrome by the menthol test. Acta Paediatr. Hung. 4:153.

9. Arias, I. M., L. M. Gartner, M. Cohen, J. Ben Ezzer, and A. J. Levi. 1969. Chronic non-hemolytic unconjugated hyperbilirubinemia with glucuronyl transferase deficiency: clinical, biochemical, pharmacologic, and genetic evidence for heterogeneity. Am. J. Med. 47:395-409.

10. Ritter, J. K., F. Chen, Y. Y. Sheen, H. M. Tran, S. Kimura, M. T. Yeatman, and I. S. Owens. 1992. A novel complex locus UGTI encodes human bilirubin, phenol, and other UDP-glucuronosyltransferase isozymes with identical carboxyl termini. J. Biol. Chem. 267:3257-3261.

11. Harding, D., S. J. Jeremiah, S. Povey, and B. Burchell. 1990. Chromo- 
somal mapping of a human phenol UDP-glucuronosyltransferase, GNT1. Ann. Hum. Genet. 54:17-21.

12. Roy Chowdhury, J., N. Roy Chowdhury, G. Wu, R. Shouval, and I. M. Arias. 1981. Bilirubin monoglucuronide and diglucuronide formation by human liver in vitro assay by high pressure liquid chromatography. Hepatology. 1:622627.

13. Roy Chowdhury, J., N. Roy Chowdhury, U. Gartner, A. W. Wolkoff, and I. M. Arias. 1982. Bilirubin diglucuronide formation in intact rats and in isolated Gunn rat livers. J. Clin. Invest. 69:595-603.

14. Hanahan, D. 1985. Techniques for transformation of E. Coli. In DNA Cloning Volume I: A Practical Approach. D. M. Glover, editor. IRL Press, Washington, DC. 109-135.

15. Ritter, J. K., Y. Y. Sheen, and I. S. Owens. 1990. Cloning and expression of human liver UDP-glucuronosyltransferase in COS-1 cells. 3,4-Catechol estrogens and estriol as primary substrates. J. Biol. Chem. 265:7900-7906.

16. Roy Chowdhury, N., P. Gross, A. D. Moscioni, M. Kram, I. M. Arias, an J. Roy Chowdhury. 1987. Isolation and purification of multiple normal and functionally defective forms of UDP-glucuronosyltransferase from livers of inbred Gunn rats. J. Clin Invest. 79:327-334.

17. Harding, D., S. Fournel-Gigleux, M. R. Jackson, and B. Burchell. 1988. Cloning and substrate specificity of a human phenol UDP-glucuronosyltransferase expressed in COS-7 cells. Proc. Natl. Acad. Sci. USA. 85:8381-8385.

18. Keen, J., D. Lester, C. Inglehearn, A. Curtis, and S. Bhattacharya. 1991 Rapid detection of single base mismatches as heteroduplexes on Hydrolink ${ }^{\mathrm{TM}}$ gels. Trends Genet. 7:5.

19. Dutton, G. J. 1980. Glucuronidation of Drugs and Other Compounds. CRC Press, Boca Raton, FL. 3-78.

20. van Es, H. H. G., B. G. Goldhoorn, M. Paul-Abrahamse, R. P. J. Oude Elferink, and P. L. M. Jansen. 1990. Immunochemical analysis of uridine diphosphate-glucuronosyltransferase in four patients with the Crigler-Najjar Syndrome Type I. J. Clin. Invest. 85:1199-1205.

21. Tephly, T. R., and B. Burchell. 1990. UDP-glucuronosyltransferase: a family of detoxifying enzymes. TIPS (Trends Pharmacol. Sci.) 11:276-279.

22. Sato, H., O. Koiwai, K. Tanabe, and S. Kashiwamata. 1990. Isolation and sequencing of rat liver bilirubin UDP-glucuronosyltransferase cDNA: possible alternate splicing of a common primary transcript. Biochem. Biophys. Res. Commun. 169:260-264.
23. Ritter, J. K., F. Chen, Y. Y. Sheen, R. A. Lubet, and I. S. Owens. 1992. Two human liver cDNAs encode UDP-glucuronosyltransferase with 2 log differences in activity toward parallel substrates including hyodeoxycholic acid and certain estrogen derivatives. Biochemistry. 31:3409-3414.

24. Schmid, R., J. Axelrod, L. Hammaker, and R. L. Swarm. 1958. Congenital jaundice in rats due to a defective glucuronide formation. J. Clin. Invest. 37:1123-1130.

25. Iyanagi, T., T. Watanabe, and Y. Uchiyama. 1989. The 3-methylcholanthrene-inducible UDP-glucuronosyltransferase deficiency in the hyperbilirubinemic rat (Gunn rat) is caused by a -1 frameshift mutation. J. Biol. Chem. 264:21302-21307.

26. Iyanagi, T., M. Haniu, K. Sogawa, Y. Fujii-Kuriyama, S. Watanabe, J. E. Shively, and K. F. Anan. 1986. Cloning and characterization of cDNA encodin 3-methylcholanthrene inducible rat mRNA for UDP-glucuronosyltransferase. $J$ Biol. Chem. 261:15607-15614.

27. ElAwady, M., J. Roy Chowdhury, K. Kesari, H. Van Es, P. L. M. Jansen M. Lederstein, I. M. Arias, and N. Roy Chowdhury. 1990. Mechanism of the lack of induction of UDP-glucuronosyltransferase activity in Gunn rats by 3-methylcholanthrene. Identification of a truncated enzyme. J. Biol. Chem. 265:1075210758.

28. Sato, H., S. Aono, S. Kashiwamata, and O. Koiwai. 1991. Genetic defect of bilirubin UDP-glucuronosyltransferase in the hyperbilirubinemic Gunn rat. Biochem. Biophys. Res. Commun. 177:1161-1164.

29. Watkins, J. B., and C. D. Klaassen. 1982. Induction of UDP-glucuronosyltransferase activities in Gunn, heterozygous, and wistar rat livers by pregnenolone-16 $\alpha$-carbonitrile. Drug Metab. Dispos. 10:590-594.

30. Abe, N., E. Abe, and A. Yuasa. 1988. Purification and properties of 5-hydroxytryptamine UDP-glucuronyltransferase from rat liver microsomes. J. Biochem. 104:421-426.

31. De Morais, S. M. F., and P. G. Wells. 1988. Deficiency in bilirubin UDPglucuronyltransferase as a genetic determinant of acetaminophen toxicity. $J$. Pharmacol. Exp. Ther. 247:323-331.

32. Robertson K. J., D. Clarke, L. Sutherland, R. Wooster, M. W. H. Coughtrie, and B. Burchell. 1991. Investigation of the molecular basis of the genetic deficiency of UDP-glucuronosyltransferase in Crigler-Najjar syndrome. $J$. Inherited Metab. Dis. 14:563-579. 